\title{
Seizures after intravenous contrast media for cranial computed tomography
}

\author{
MICHAEL NELSON, RICHARD J V BARTLETT, JOHN T LAMB \\ From the General Infirmary, Leeds, UK
}

SUMMARY Patients who had contrast medium-induced seizures were reviewed retrospectively in a consecutive series of 15,226 contrast-enhanced head CT examinations. An incidence of $0 \cdot 19 \%$ was found. There was a strong association with a history of spontaneous seizure and with the presence of a structural intracranial abnormality. The contrast medium-induced seizures were short-lived and selflimiting or readily controlled with small doses of intravenous diazepam. They were clinically benign.

Seizures are rare after intravenous injection of iodinated radiographic contrast media. In patients undergoing excretory urography an incidence of $0.001-$ $0.01 \%$ has been reported..$^{1-3}$

Patients who have computed tomography (CT) of the brain might be expected to have a higher incidence of seizure because epilepsy and structural lesions which lower the seizure threshold are more common in this selected group. An incidence of $0.5 \%$ was found in two series. ${ }^{45}$ The risk of seizure is particularly high in patients with cerebral metastatic disease undergoing contrast-enhanced CT, in whom an incidence of 6 $19 \%$ is reported. ${ }^{4-6}$

In the General Infirmary at Leeds we have used a greater variety of contrast media in a wider range of osmolalities and doses than has been previously reported in series of contrast-enhanced cranial CT examinations.

\section{Methods}

The records of the CT Head Scanning Department from September 1976 to August 1987 were examined to identify patients who developed seizures after intravenous contrast medium and to establish the overall pattern of contrast medium usage. An event was defined as a seizure if it was an episode of paroxysmal uncontrolled motor activity, focal or generalised, or a period of "absence" or a paroxysmal focal sensory disturbance. Patients were included in the survey only if they were conscious and not in a post-ictal state when

Address for reprint requests: Dr M Nelson, Department of Diagnostic Radiology, The General Infirmary at Leeds, Great George Street, Leeds LSI 3EX, UK.

Received 15 July 1988 and in revised form 31 March 1989. Accepted 7 May 1989 contrast medium was injected and if seizure started less than 30 minutes after injection. Seizure incidence was calculated for different doses of each contrast medium and confidence limits were estimated assuming a Poisson distribution. Fisher's exact two-tailed test was used to assess the significance of the variations in seizure incidence.

The technique of contrast medium injection varied little between patients. For adults a 19 gauge butterfly needle was inserted in an arm vein and contrast medium was injected as rapidly as possible by hand, in practice taking 2-3 minutes for injections of $100 \mathrm{ml}$. After injection patients were observed continuously for 30 minutes by a senior radioo grapher. In the event of a seizure a radiologist was called promptly to attend the patient. Seizures were treated witbo intravenous diazepam (Valium, Roche) at the discretion of the attending radiologist.

The patients' medical records and CT scans were reviewed to determine; (1) the type and duration of the contrast medium-induced seizure; the dose and concentration of the contrast medium used; the time of seizure onset after contrast injection and whether the seizure was self-limited or lasted sufficiently long to require treatment, in the view of attending medical staff. (2) The type and duration of previous seizures. (3) Anticonvulsant therapy at the time of CT scan. (4) Previous or concurrent brain radiotherapy or chemotherapy. (5) Adverse reactions to previous or subsequent contrast medium injections. (6) Intracranial pathology at the time of seizure and associated CT abnormalities, particularly the presence of cerebral oedema and pathologic contrast enhancement.

\section{Results}

In the survey period of 11 years 29,100 cranial CT examinations were performed on 20,305 patients. Intravenous enhancement was used for 15,226 $(52.3 \%)$ examinations. Twenty nine patients had contrast medium-induced seizures, an incidence of $0 \cdot 19 \%$ 


\section{Contrast media and seizure incidence}

The contrast media which were used in adults are listed by iodine content and volume administered in table 1 with the number of examinations and the associated number and incidence of seizures. With the two ionic contrast media, iothalamate and metrizoate, seizures occurred at all concentrations $(280-420 \mathrm{mg} / \mathrm{ml}$ iodine content) and with "single" and "double" dose examinations (approximately 20 and $40 \mathrm{gm}$ iodine respectively). There were no seizures with the non-ionic contrast medium iopamidol in 1169 examinations. A single case of seizure occurred using iohexol in 1571 examinations.

Because seizure is a rare event in statistical terms, the values of seizure incidence specified in table 1 are unlikely to reflect accurately any real differences in the toxicity of the contrast media. The overall adult incidence rate for data in table 1 of 0.18 was used to calculate the expected number of seizures using the different contrast media. Ninety five per cent confidence limits were also calculated for these expected numbers of seizures under the null hypothesis of a constant seizure rate, irrespective of medium. Examination of the table shows that only the $100 \mathrm{ml}$ dose of meglumine metrizoate and the $50 \mathrm{ml}$ dose of sodium iothalamate have observed numbers of seizures on or outside these confidence limits. For several contrast media no lower limit can be calculated, because of the extreme rarity of the events, and hence the lack of information to base any estimates on.

Fisher's Exact test was applied to three comparisons of interest. There was a higher incidence of seizure with $100 \mathrm{ml}$ of either ionic medium than with $50 \mathrm{ml}$ of sodium iothalamate (with sodium iothalamate $100 \mathrm{ml}$ (p < 0.05), with meglumine metrizoate $100 \mathrm{ml}(\mathrm{p}<$ $0.001)$ ). With $50 \mathrm{ml}$ of meglumine metrizoate there was a higher incidence of seizure than with $50 \mathrm{ml}$ of sodium iothalamate $(p<0.01)$.

Contrast medium-induced seizures were observed in three children under 10 years. The incidence of $0.36 \%$, in 556 patients given meglumine iothalamate
$280 \mathrm{mgI}_{2} / \mathrm{ml}$ in a dose of $2 \mathrm{ml} / \mathrm{kg}$ body weight, was similar to that in adults who had "double" dose examinations. One child had a seizure after administration of sodium iothalamate. There were no seizures in 186 children who received non-ionic media.

\section{Patients and seizure history}

Contrast medium-induced seizures occurred in children and adults between the ages of 6 and 78 years, mean age 49 years. Fourteen patients were female, 15 male. Of the 29 patients who had contrast mediuminduced seizures $22(76 \%)$ were known to have had previous spontaneous seizure.

A history of some craniocerebral disorder or therapy which might cause seizure was known in 26 patients; some had multiple risk factors. Twenty-two patients had a history of previous seizure; seven of intracranial tumour and five of head injury. Seven patients had previous craniotomy and five had radiotherapy. The seven patients with intracranial tumour comprised five with cerebral glioma, all of whom were known to have residual tumour, and two patients with previously excised supratentorial meningioma, one of which was recurrent. The five cases of craniocerebral radiotherapy received treatment between three months and ten years before the contrast medium-induced seizure. Two patients were receiving treatment with epileptogenic drugs; imipramine, thioridazine and dothiepin in one patient; prochlorperazine maleate in the other. Two patients with epilepsy, on sodium valproate, had contrast mediuminduced seizures within one month of a dose reduction.

In those with seizures a clinical presentation suggesting metastatic disease was present in seven patients. Two had proven breast carcinoma, three primary bronchial carcinoma, and one had carcinoma of the colon. One patient had skin metastases from an unknown primary source.

In the 22 patients who had previous "spontaneous" seizures the length of history prior to their contrast

Table 1 Contrast medium-induced seizures in adults. Incidence and statistical analysis

\begin{tabular}{|c|c|c|c|c|c|c|}
\hline Contrast Medium & $m l$ & $\begin{array}{l}\text { Number of } \\
\text { examinations }\end{array}$ & Seizures & Incidence & $\begin{array}{l}\text { Expected } \\
\text { number } \\
\text { of seizures }\end{array}$ & $\begin{array}{l}95 \% \text { confidence } \\
\text { limits for the } \\
\text { expected number } \\
\text { of seizures }\end{array}$ \\
\hline $\begin{array}{l}\text { Sodium } \\
\text { Iothalamate } \\
\left(420 \mathrm{mgI}_{2} / \mathrm{ml}\right) \\
\text { Meglumine } \\
\text { Metrizoate } \\
\left(370 \mathrm{mgl}_{2} / \mathrm{ml}\right) \\
\text { lopamidol } \\
\left(300 \text { or } 370 \mathrm{mgI}_{2} / \mathrm{ml}\right) \\
\text { Iohexol } \\
\left(300 \text { or } 350 \mathrm{mgI}_{2} / \mathrm{ml}\right) \\
\text { Total }\end{array}$ & $\begin{array}{r}100 \\
50 \\
<50 \\
100 \\
50 \\
<50 \\
100 \\
50 \\
100 \\
50\end{array}$ & $\begin{array}{r}382 \\
4491 \\
423 \\
6098 \\
268 \\
82 \\
980 \\
189 \\
805 \\
766 \\
14484\end{array}$ & $\begin{array}{r}2 \\
1 \\
1 \\
18 \\
3 \\
0 \\
0 \\
0 \\
0 \\
1 \\
26\end{array}$ & $\begin{array}{l}0 \cdot 52 \\
0 \cdot 01 \\
0 \cdot 24 \\
0 \cdot 29 \\
1 \cdot 12 \\
0 \\
0 \\
0 \\
0 \\
0 \cdot 13 \\
0 \cdot 18\end{array}$ & $\begin{array}{r}0.7 \\
8 \cdot 1 \\
0 \cdot 8 \\
11 \cdot 0 \\
0.5 \\
0 \cdot 1 \\
1 \cdot 8 \\
0.3 \\
1.4 \\
1.4 \\
26\end{array}$ & $\begin{array}{l}0-2 \\
2-14 \\
0-2 \\
4-18 \\
0-2\end{array}$ \\
\hline
\end{tabular}


induced seizure varied from less than a day to 18 years.

Of eight patients with a history of less than one week, two presented after a recent head injury, one with an encephalitic illness, and one was suffering acute alcohol withdrawal; the other four were found to have intracranial mass lesions (two primary, two secondary). All five patients who had a history of seizure lasting between one week and six months had cerebral neoplasia; four had secondary deposits from known primary carcinomas and one had a high grade glioma. None of the nine patients with a history longer than six months had any clinical or radiological evidence of metastatic disease. The pre-existing seizures showed various clinical patterns. They were grand mal in nine patients, focal motor in eight, combined grand mal and Jacksonian focal in four and combined grand mal and temporal lobe in one. Patients with mixed patterns of seizure all had longstanding seizures.

\section{Contrast medium-induced seizures}

The clinical patterns of contrast-induced seizures are listed in table 2. No patient had status epilepticus. Ten patients $(34 \%)$ had partial seizures and $19(66 \%)$ had grand mal seizures. Of the 15 patients who had previous grand mal seizures 11 had grand mal seizures after contrast. An adult who had previous grand malJacksonian seizures had a focal motor seizure; another who had previous grand mal seizures had a focal motor episode consisting of transient aphasia and facial palsy. A six year old child with previous grand mal, focal motor and temporal lobe seizures had an episode of absence of petit mal type. Of the eight patients who had previous focal motor seizures, four had similar seizures after contrast and four had grand mal seizures. A 56 year old woman with a history of breast carcinoma, hypertension and a small stroke that left her with a mild right hemiparesis had an episode of severe and distressing pain in the left leg after injection of $50 \mathrm{ml}$ Conray $420 \mathrm{mgI}_{2} / \mathrm{ml}$ via an arm vein. The pain resolved spontaneously in less than five minutes and CT showed mild atrophy and no focal lesion. There has been no similar episode or evidence of symptomatic cerebrovascular disease in ten years follow up. While the description of the acute episode is compatible with transient ischaemia or focal sensory epilepsy, we incline to the latter interpretation because of the patient's pronounced emotional distress, the

Table 2 Clinical patterns of contrast-induced seizures

\begin{tabular}{lcl}
\hline & Previous fits & No previous fits \\
\hline Grand mal & 15 & 4 \\
Focal motor & 6 & 2 \\
Focal sensory & 1 & 1 \\
Petit mal & & \\
\hline
\end{tabular}

Table 3 CT findings at the time of contrast medium-induced seizure (29 patients)

\begin{tabular}{ll}
\hline CT findings & $\begin{array}{l}\text { Number } \\
\text { of patients }\end{array}$ \\
\hline Normal & 4 \\
Generalised "atrophy" & 1 \\
Focal abnormality & 24 \\
Intracranial mass & 18 \\
Focal atrophy, infarction or post surgical change & 5 \\
Calcification of inferior vermis & 1
\end{tabular}

close temporal relationship between the attack and contrast injection and the lack of relevant focal symptoms with long follow up.

The timing of seizures in relation to the injection of contrast was variable. Onset of seizure during injection was uncommon, occurring in only two patients, but $24(83 \%)$ cases occurred within ten minutes; five cases occurred between eleven and thirty minutes. The length of seizure history and the clinical pattern of previous seizures bore no simple relationship to the timing.

The contrast medium-induced seizures lasted less than five minutes in 27 patients $(93 \%)$. A 67 year old man had a grand mal seizure which lasted 10 minutes and resolved without anticonvulsant medication Another patient had a similar duration of grand mad seizure despite treatment with $30 \mathrm{mg}$ of diazepan intravenously. A 64 year old woman with a history of grand mal seizures had a five minute grand mal seizurê which started about 15 minutes after the injection of $50 \mathrm{ml}$ iohexol $300 \mathrm{mgI}_{2} / \mathrm{ml}$; this resolved spontane eously but was followed by a series of brief grand maf seizures during the next thirty minutes. The seizures resolved spontaneously in 20 patients $(69 \%)$. Intravenous diazepam was given to nine patients during the seizure $(31 \%)$ in doses ranging from $5-30 \mathrm{mg}$.

Fourteen patients were taking anticonvulsant medication at the time of the contrast mediuminduced seizure. Plasma levels of either phenytoin or carbamazepine were within the therapeutic range in the only four patients whose levels were measured less than three months before the contrast mediuminduced seizure.

Sixteen patients had contrast-enhanced CT examinations on 30 occasions before or after the contrast medium-induced seizure. Nine patients received the contrast medium which produced their seizure in the same or higher dose on 15 other occasions; none of these examinations resulted in seizure. The single patient who had a seizure after the non-ionic contrast medium iohexol, subsequently received an ionic contrast medium on two other occasions without seizure.

There was only one patient in whom previous exposure to contrast media had induced a fit. A 22 year old woman had a four year history of poorly con. o 
trolled grand mal epilepsy with several episodes of status epilepticus. Two years previously she had a seizure induced by $50 \mathrm{ml}$ of sodium iothalamate at another hospital. Her anticonvulsant dosage had been increased four days before CT scanning and she failed to reveal the history of previous seizure on direct questioning. She received $50 \mathrm{ml}$ of meglumine metrizoate and suffered a grand mal convulsion which lasted 10 minutes despite prompt treatment with $30 \mathrm{mg}$ of diazepam.

Findings on CT scan at the time of the contrast medium-induced seizure are indicated in table 3. Four patients had normal scans, though all had previous seizures and presumptive evidence of a neurological lesion, after head injury in two cases, during an encephalitic illness in a third, and with long standing poorly controlled epilepsy in another. One patient with acute alcohol withdrawal had generalised atrophy.

The remaining twenty-four patients had focal CT abnormalities. Eighteen had one or more intracranial mass lesions; five had focal atrophy, infarction or post-surgical change; one had calcification in the cerebellar vermis, the nature of which remains uncertain. Of the 18 patients with intracranial mass lesions, seven had biopsy-proven gliomas and two had meningiomas. Six patients had a proven primary carcinoma with intracranial masses, solitary in three and multiple in three. Three patients had unbiopsied solitary cerebral lesions which were thought to be gliomas.

Although it is difficult to determine accurate incidence values from our data, it is our impression that in our series the incidence of contrast mediuminduced seizures is similar in patients with metastatic disease and with non-metastatic intracranial tumours.

Pathological contrast enhancement occurred in 15 of the patients with mass lesions on CT. Three gliomas did not demonstrate enhancement. The site of all solitary mass lesions is plotted in the figure; most are located in the frontal and parietal lobes.

\section{Discussion}

The incidence of seizures after intravenous contrast enhancement for computed tomography of the brain is likely to depend on the presence of cranio-cerebral pathology and on the physical and chemotoxic properties of contrast media. The present study agrees with previous authors ${ }^{45}$ that the incidence of contrast medium-induced seizures with cranial CT $(0 \cdot 2-0 \cdot 5 \%)$ is considerably higher than that associated with excretory urography $(0.001-0.01 \%)$. The higher incidence is likely to be due to the presence of craniocerebral pathology in patients who undergo cranial CT.

We have found a variety of predisposing factors and cranio-cerebral pathologies in association with con-
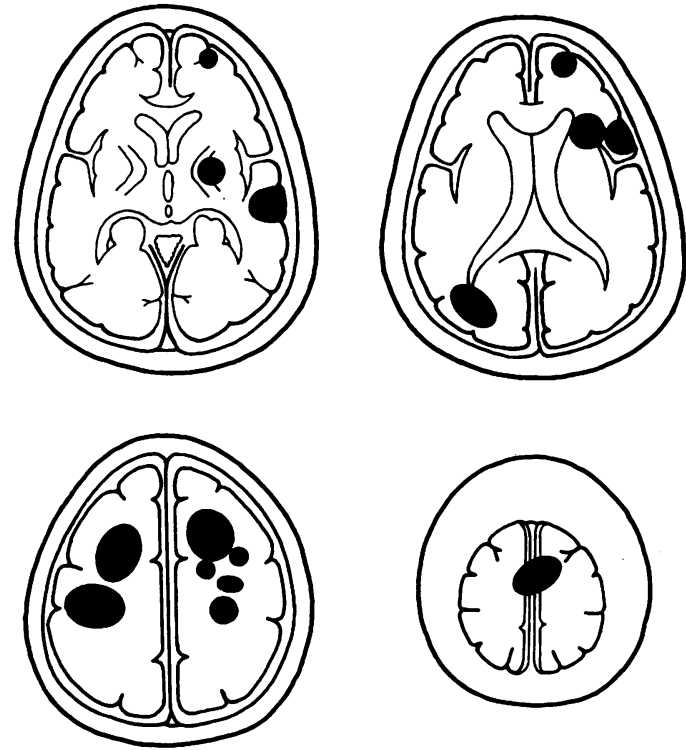

Fig Location of solitary mass lesions on CT (15 patients).

trast medium-induced seizures. Focal pathology was apparent in most of our cases. The five patients who did not have a focal abnormality on CT all had previous seizures, which were focal in two. There were similar findings in the two previous unselected series of contrast medium-induced seizures. All five of Lo Zito's patients had clinical and CT evidence of focal cerebral pathology ${ }^{4}$ and all seven of Scott's patients had either clinical or CT evidence of focal pathology. ${ }^{5}$

The high incidence of previous seizures in our cases $(76 \%)$ and in Pagani's cases with cerebral metastasis (13 out of 17 patients, $76 \%)^{6}$ suggests that contrast medium-induced seizures occur in the presence of an overt or latent epileptogenic focus.

It seems unlikely that contrast media could precipitate seizures without coming into contact with neural tissue. Pathological contrast enhancement was observed in the intracerebral lesions of $15(52 \%)$ of our patients; a further eight $(28 \%)$ had focal intracranial pathologies which one would reasonably expect to be accompanied by increased permeability of the bloodbrain barrier. Six $(21 \%)$ patients had intracranial pathologies which seemed unlikely to be associated with impairment of the blood-brain barrier. In such cases, extravasation of contrast medium may depend on foci of increased blood-brain barrier permeability secondary to previous seizure activity ${ }^{7}$ or on the mechanisms by which small amounts of contrast medium enter the cerebral interstitial space or cerebrospinal fluid after intravenous injection in the absence of cerebral pathology ${ }^{89}$ 
Intracranial pathology with mass effect, present in $18(62 \%)$ of our patients, produces local slowing of the cerebral circulation. It has been suggested that this results in a prolonged exposure of the vascular endothelium to the high levels of intra-arterial contrast medium which occur shortly after intravenous injection and thereby allows increased extravasation into the brain. ${ }^{10}$

In previously reported series patients with cerebral metastasis have been found to be particularly at risk of contrast medium-induced seizures. ${ }^{46}$ This might be anticipated from their frequent presentation with focal cerebral lesions that show mass effect and prominent contrast enhancement. In comparison, only six (21\%) of our patients had actual or suspected cerebral metastasis, considerably fewer than we would have expected from the number of patients with known metastatic disease examined during the survey, though we have not obtained accurate data to quantitate this. The difference may be accounted for by differences in the dose of contrast medium. The higher incidence of seizures in those series which used larger doses of contrast medium ${ }^{46}$ suggests that seizure incidence in patients with metastatic disease may be dose-dependent.

From previous studies it is not clear whether the incidence of seizure is related to the chemical composition of the contrast medium. Lo Zito encountered seizures with sodium iothalamate and meglumine diatrizoate but did not provide sufficient data for statistical analysis. Scott ${ }^{5}$ used the same dose of a single contrast medium in all patients. Pagani et $a^{6}$ state that the risk of seizure is not related to the chemical composition of contrast media but provide no data to support this since all their patients received a single agent, meglumine diatrizoate.

In our experience contrast medium-induced seizures were a benign entity. Most lasted less than five minutes $(93 \%)$ and resolved spontaneously $(69 \%)$. In the patients who required anticonvulsant medication only a small dose of intravenous diazepam was necessary to terminate the seizure. The pattern of the seizure was benign in that status epilepticus did not occur and there were partial seizures in $34 \%$ of cases. Anxiety was minimised to some extent by the fact that $76 \%$ of patients had experienced seizures previously. It was convenient for the management of patients that seizure developed shortly after contrast injection, at which time the patients were under close observation in the scanning department where full facilities for resuscitation were available.

Our findings have implications for our policy on contrast medium usage during CT scanning. Seizures occurred after ionic and non-ionic contrast media and seizure incidence did not correlate simply with the dose of contrast medium. On these grounds there is no strong indication to use any particular contrast medium or to limit the dose. In our practice the exclusive use of non-ionic contrast media would be prohibitively expensive and we restrict their use to patients who are especially at risk. For patients without specific risk factors an ionic contrast medium may be considered adequate and combines low cost with a low risk of seizure. Limiting the dose would further reduce costs and might reduce the already small risk of seizure.

A different approach to the problem of reducing seizure risk has been taken by Pagani et al.$^{6}$ In patients with cerebral metastases they found that $5 \mathrm{mg}$ of intravenous diazepam administered immediately before contrast medium reduced the incidence of contrast medium-induced seizure by a factor of $\mathbf{0 . 2 6}$. Seventeen patients had contrast medium-induced seizures and all but one were successfully treated with varying amounts of intravenous diazepam. While the immediate expense of this approach is considerably less than that of using a non-ionic contrast medium, additional costs can be anticipated as a result of adverse reactions to the use of diazepam. It is not yet known whether diazepam reduces the incidence of contrast medium-induced seizures associated with non-metastatic intracranial pathologies.

Our view is that the rarity of contrast mediuminduced seizures, the benign short-lived self-limiting nature of most seizures and the prompt response of seizures to intravenous diazepam together justify the choice of contrast agent and its dose on the basis of cost and/or clinical considerations apart from the risk of seizure. The expense of using non-ionic media or diazepam prophylaxis in the large number of patients who are at risk of seizure is not warranted.

We thank Dr A D Clayden for statistical advice, and Mrs Y P Wright for secretarial assistance.

\section{References}

1 Ansell G. Adverse reactions to contrast agents. Invest Radiol 1970;5:374-84.

2 Wolfromn R, Dehouve A, Degand F, Wattez E, Crehalet $A$. Les accidents graves par injection intraveineuse de substances iodées pour urographie. Journal de Radiologie et d'Electrologie 1966;47:346-57.

3 Witten DM, Hirsch FD, Hartman GW. Acute reactions to urographic contrast medium. Am J Roentgenol 1973;119:832-40.

4 Lo Zito JC. Convulsions: a complication of contrast enhancement in computerized tomography. Archives Neurol 1977;34:649-50.

5 Scott WR. Seizures: a reaction to contrast media for computed tomography of the brain. Radiology 1980; 137:359-61.

.


6 Pagani JJ, Hayman LA, Bigelow RH, Libshitz HI, Lepke RA, Wallace S. Diazepam prophylaxis of contrast media-induced seizures during computed tomography of patients with brain metastases. Am J Neuroradiol 1983;4:67-72.

7 Dillon W, Brant-Zawadzki M, Sherry RG. Transient computed tomography abnormalities after focal seizures. Am J Neuroradiol 1984;5:107-9.
8 McClennan BL, Becker JA. Cerebrospinal fluid transfer of contrast material at urography. Am J Roentgenol 1971;113:427-32.

9 Sage MR. Kinetics of water-soluble contrast media in the central nervous system. Am J Neuroradiol 1983;4: 897-906.

10 Fischer HW. Occurrence of seizure during cranial computed tomography. Radiology 1980;137:563-4. 